\title{
Quaderni
}

QUADERNI Communication, technologies, pouvoir

73 | Automne 2010

La métropole parisienne entre récits, paroles et échanges

\section{La métropole parisienne au prisme du réseau : réalités discursives et marqueurs symboliques}

\section{Gustavo Gomez-Mejia}

\section{OpenEdition}

Journals

Édition électronique

URL : http://journals.openedition.org/quaderni/447

DOI : $10.4000 /$ quaderni.447

ISSN : 2105-2956

Éditeur

Les éditions de la Maison des sciences de l'Homme

Édition imprimée

Date de publication : 5 octobre 2010

Pagination : 53-64

Référence électronique

Gustavo Gomez-Mejia, «La métropole parisienne au prisme du réseau : réalités discursives et marqueurs symboliques », Quaderni [En ligne], 73 | Automne 2010, mis en ligne le 05 octobre 2012, consulté le 01 mai 2019. URL : http://journals.openedition.org/quaderni/447 ; DOI : 10.4000/ quaderni.447 


\section{$D$ ossier}

\section{la métropole parisienne au prisme du réseau :} réalités discursives et marqueurs symboliques

\section{Gustavo Gomez-Mejia}

Doctorant en Sciences de l'Information et de la Communication

CELSA, GRIPIC Université Paris Sorbonne
En tant qu' espace de communication à vocation globale, Internet a accueilli depuis ses origines les écritures et les débats de projets divers, individuels et collectifs. Le projet d'une métropole parisienne s'inscrit dans la lignée d'autres projets que le réseau alimente et accompagne dans un spectre discursif qui va des fantasmes utopiques aux propositions exécutables. Nous nous proposons de faire un état des lieux des formes dominantes de représentation de la métropole parisienne sur Internet aujourd'hui, à partir d'un corpus de sites Web (institutionnels, médiatiques, forums et réseaux sociaux). D'un point de vue communicationnel, il s'agit d'analyser les intérêts, langages et techniques de différentes discursivités qui, dans leur polyphonie, dessinent des zones de rencontre mais aussi des écarts autour de la métropole en devenir. Des domaines territoriaux aux centralités métamédiatiques du Web contemporain, nous interrogeons les réalités discursives que coproduisent les dispositifs et les acteurs dans leur rapport aux différents marqueurs symboliques qui font métropole. Comment circulent ces discours sur la métropole parisienne? Quelles sont les formes qu'ils adoptent dans leur médiatisation en ligne? Projets politiques, retombées médiatiques, cautions d'experts et paroles vernaculaires configurent cet espace problématique dont nous explorons les logiques sociales et les formes d'expression.

Les constructions discursives de la Métropole : des écarts ou des éclats?

Tel que le rappelle Eliseo Véron, «l'analyse des discours ne peut travailler que sur des écarts inter-discursifs, elle est toujours inter-discursive $»^{1}$. Dès lors que ces discursivités se matérialisent sur 
des sites Web produits par des énonciateurs aux intérêts divers, cette vaste production de sens sur le projet métropolitain reflète des fonctionnements sociaux complexes et plurivoques. $\mathrm{Si}$ la métropole parisienne existe dans ces discours qui circulent sur Internet, il semble alors légitime de se demander : d'où est-elle pensée ? Dans la continuité de l'état des lieux sur «l'inscription $d u$ projet de Métropole de Paris dans l'espace public numérique $»^{2}$ mené par Dominique Pagès, c'est donc un réseau de distances inter-discursives qui se donne à lire en fonction de la reconnaissance de certains marqueurs constitutifs du discours métropolitain par les acteurs impliqués. Trois grands axes de circulation du discours se dessinent, créant des alliances plus ou moins perméables qui vont des institutions aux habitants.

Des énonciateurs publics aux récits médiatiques : rouages institutionnels du discours " métropolitisé "

La genèse institutionnelle d'un questionnement politique sur l'avenir de Paris marque fortement, dès l'année 2001, les conditions de production des discours sur le devenir métropolitain. L'énonciation d'un discours sur la métropole parisienne prend ainsi la forme dominante du 'projet politique'. À cet égard, la délégation de l'élu Pierre Mansat comme adjoint au projet qui aboutira à la création de la Conférence métropolitaine en 2006, puis à l'établissement du syndicat mixte « Paris Métropole » en 2009, présente une homologie formelle avec la nomination en 2008 de Christian Blanc au poste de secrétaire d'État chargé du Développement de la région capitale à l'origine du projet du « Grand Paris » ${ }^{3}$. D'un point de vue discursif, ce sont deux projets politiques qui articulent chacun un réseau d'énonciateurs publics autour d'une réalité métropolitaine dont le sens reste à produire, et dont l'action est en quête de légitimité et d'efficacité. Pour parler comme Michel de Certeau, le point commun reste celui qui manifeste dans les discours une prétention « stratégique » à parler de la métropole comme d'un « lieu propre $»^{4}$. La rationalité politique dessine alors une métropole vue d'en haut, dont la perspective hésite toutefois entre les points de vue de plusieurs échelons territoriaux : un État commanditaire? Une gouvernance de la Région? La Capitale Paris à la pointe « locomotive » de sa Banlieue ? La mise en discours des genèses des deux projets traduit bien ces décalages territoriaux et ressource les visions métropolitaines à différents niveaux.

Sur le site « Le Grand Paris » ${ }^{5}$, créé en novembre 2009 par le Service d'Information du Gouvernement, « le 29 avril » 2009 est construit comme moment initiatique : date à laquelle « le président de la République exposait son ambition pour le Grand Paris de demain à la Cité de l'Architecture et $d u$ Patrimoine $»^{6}$. Le discours présidentiel est mis en exergue comme « pourquoi » de la « consultation » : «Le Grand Paris, c'est un projet qui concerne tout le monde et qui appartient à tout le monde " ${ }^{7}$. Dans "cette nouvelle page de l'histoire de Paris », la construction de «la villemonde de demain » ou de «la métropole durable de l'après Kyoto » œuvre comme corollaire d'une discursivité déployant la production des «dix équipes d'architectes internationaux ».

Sur le site de $«$ Paris Métropole $»^{8}$, cette genèse affirme davantage une prétention à l'ancienneté et à la collectivité comme marqueurs légitimants. 
« La création de Paris Métropole s'inscrit dans une démarche enclenchée par des élus de l'agglomération parisienne dès $2001 »$ affirme le texte de présentation «De la conférence métropolitaine à Paris Métropole » : il est question de décrire la trajectoire d'un projet ponctué de dates et de moments quantifiables : « 400 acteurs », « 150 élus », la conférence qui «s'est réunie à 12 reprises » entre 2006 et 2009.

Entre ces deux sites correspondant aux projets politiques principaux, deux registres se développent allant des visions étatiques aux regards intercommunaux : la projection des symboles forts d'une capitale agrandie fait ainsi face à la complexe logistique métropolitaine qui se met en place, laquelle reste moins apte à l'ethos d'une allocution qu'au détail d'un compte-rendu. D'un point de vue discursif, la métropole comme territoire entretient donc les clivages politiques et les décalages d'échelons de ses énonciateurs : face au constat d'un discours politiquement clivé, le choix d'une reprise énonciative des syntagmes « Grand Paris » ou « Paris Métropole » vaut alors 'reconnaissance' d'un projet ou de l'autre et scinde les représentations métropolitaines.

Sur le site du «Conseil régional d'île-deFrance $»{ }^{9}$, échelon territorial mitoyen, les deux dénominations coexistent : le moteur de recherche du site recense 98 occurrences pour « Grand Paris » et 174 pour « Paris métropole », lesquelles indexent des documents publiés autour des réactions de Jean-Pierre Huchon. À la manière d'un hapax, une co-occurrence spéciale qui voit juxtaposés les noms des deux projets renvoie à la description du poste de « Jean-Luc Laurent, Délégué spécial auprès du Président sur le Grand
Paris et sur la métropole francilienne. Représentant du Président à Paris Métropole $»^{10}$.

Dans cette mise en discours institutionnelle qui voit la métropole parisienne scindée entre les projets et leurs hommes, la reprise d'une dénomination ou d'une autre tisse sur la toile des réseaux discursifs distincts. En dehors des sites consacrés aux projets eux-mêmes, le mot-clé " Grand Paris » renvoie sur Google au site « Le Grand Paris $»^{11}$ lancé en 2008 par le ministère de la Culture, ou au blog $«$ Le Grand Paris $»^{12}$ du sénateur Philippe Dallier sur les serveurs du Sénat. Quant à " Paris métropole », les liens affichés pointent vers le dossier thématique que la Mairie de Paris consacre au syndicat du même nom ${ }^{13}$, ou vers « Paris métropole fédérée $»^{14}$, blog tenu par Pierre Mansat. Au-delà des affinités politiques et des circonscriptions territoriales, la bifurcation informatique de ces réseaux discursifs dépend de la saisie des mots discriminants « grand» ou « métropole ». " Combien de tâtonnements lexicaux, avant d'imposer le mot "métropole » dans le débat (zone dense, cœur d'agglo, cœur de la Région...) ? $»^{15}$ écrit Mansat sur son blog. D'un point de vue communicationnel, ces « tâtonnements » dans le « procès de dénomination » soulèvent l'enjeu décisif des formulations qui déterminent la circulation et la lisibilité d'un projet donné au prisme de Google, ainsi que ses potentielles liaisons ou cloisons dialogiques en fonction des reprises énonciatives qu'en font les acteurs ${ }^{16}$. Ces écarts entre énonciateurs plus locaux ou plus nationaux selon leurs mandats respectifs, plus à droite ou à gauche selon leurs convictions politiques, s'amplifient dès lors que l'action institutionnelle fait l'objet d'une couverture médiatique. 
Sur les sites du Monde, du Figaro, de Libération, de 20 minutes, de Rue89, du Nouvel Obs ou de $L$ 'Express, la question métropolitaine s'insère dans les espaces « politique » ou « région » des titres et bénéficie par le mécanisme des « articles liés » d'un certain « effet-rubrique $\gg{ }^{17}$ qui lui garantit une stabilité éditoriale dans l'ordre du jour. Au sein d'un discours journalistique qui reconnaît l'agenda technocratique en la réinterprétant, la médiatisation des projets tend à élaborer un récit médiatique marqué par le temps long de la prospective raconté depuis un présent conflictuel. Au sein d'un article, les co-occurrences de « Grand Paris » et de « Paris Métropole » développent la trame d'une concurrence, voire d'une agonistique. Les parallélismes formels des projets politiques favorisent ces séries d'oppositions discursives : Grand Paris vs. Paris Métropole, Droite vs. Gauche, État vs. Région, Super-métro vs. Arc Express. Tour à tour se narrativisent les actions de Blanc et de Huchon, des députés et des élus.

Du point de vue du discours médiatique, le traitement des questions propres aux instances décisionnelles (volume des budgets, prévisions infrastructurelles, événements et débats, thématisations électorales ${ }^{18}$ ) semble donc voué à accentuer une certaine 'personnalisation' des projets métropolitains, laquelle est amplifiée par l'agonistique propre au débat politique partisan. Cette première trame discursive construit la métropole parisienne sur la base d'une configuration binaire des projets politiques : les stratégies institutionnelles et leurs retombées médiatiques se reconnaissent mutuellement et anticipent la métropolisation sous le signe d'une politisation polarisante.

\section{Des experts aux architectes : accompagnements et cautions du discours métropolitain}

Face à ce premier axe inter-discursif qui - entre instances politiques et médiatiques - définit un régime dominant du projet métropolitain, il convient d'étudier un axe parallèle et perméable : celui qui conforment architectes et experts, acteurs qui jouent un rôle discursif d'accompagnement ou de caution.

Sollicités par les instances 'génétiques' des projets métropolitains, des équipes de spécialistes, d'urbanistes et d'architectes, prolongent la démarche prospective en travaillant l'adéquation des territoires actuels aux chantiers d'exécution de demain. Sur la rubrique « Métropole » du site de l'Agence Parisienne d'Urbanisme (APUR), « la construction d'une pensée métropolitaine » repose sur l'articulation des « élus » aux « professionnels de la ville $»^{19}$. Sur le site de l'Institut d'Aménagement et d'Urbanisme d'Île-de-France (IAU-IDF), il est question de « développer une pensée territoriale », de produire non «pas un dessin mais un dessein » impliquant la collaboration d' " architectes et urbanistes », « entourés de professionnels de tous horizons (économistes, paysagistes, ingénieurs...) $»^{20}$.

D'un point de vue discursif, le rôle de ces partiesprenantes du projet reste de s'ériger en figures d'experts disposant d'une visée légitimante, corrélativement perméable aux clivages politiques observés plus haut. Les études, séminaires et colloques par lesquels ces espaces en comité portent un certain « conseil scientifique » aux projets articulent ainsi les formes contemporaines du savant à la praxis technocratique. À cet égard, 
il incombe aux experts et conseillers de traduire les invariants du discours politique métropolitain en une série de visions de l'aménagement 'postparisien'. Les 29 pages de la «Biblio_GrandParis $»^{21}$ que l'IAU-IDF ressource sous le dossier «/Paris_Métropole/ » témoignent de la vitalité discursive de cet axe : la reconnaissance des impératifs de «développement durable», «mobilité 》, « compétitivité 》 et «attractivité 》au sein des rhétoriques métropolitaines du « multipolaire» ou du " polycentrique » donne lieu à un technolecte qui délimite son champ d'expertise en explorant et explosant les contours de l'«intraurbain », du «péri-urbain », du « rurbain » et $\mathrm{du}$ « méga-urbain ».

D'un point de vue idéologique, les savoirs développés par cette discursivité confortent l'inscription des projets métropolitains parisiens parmi les modélisations globalisées « d'après-Kyoto » ou dans l'émulation d'autres expériences métropolitaines (Greater London, Berlin...) dont un transfert de « bonnes pratiques »- innovant en matière d' « urbanisme » et de « gouvernance »serait à attendre. Les textes autour du colloque «Quels grands projets urbains pour l'Île-deFrance? Expériences européennes $»^{22}$, ainsi que le groupe « Projets métropolitains $~^{23}$ de l'IAU-IDF cristallisent dans le discours un mouvement de coopération et d'émulation prétendant exalter une certaine exemplarité internationale autour du cas parisien.

Là où le Schéma Directeur de la Région Île-deFrance (SDRIF) mobilise une expression textuelle et technique de ces savoirs experts ${ }^{24}$, l'expression proprement architecturale se démarque par le versant spectaculaire qu'elle favorise dans ses projections. Ainsi c'est autour de la Consultation Internationale « Le grand pari de l'agglomération parisienne $»^{25}$ que l'APUR et l'IAU-IDF ont pu surmonter décalages et échelons pour co-énoncer un discours métropolitain avec le ministère de la Culture et de la Communication. En déclinant simultanément « legrandparis.culture.gouv.fr » en « legrandparis.net » et « legrandparis.org », le site de cette instance étatique s'érige en protagoniste principal de la consultation, en même temps qu'il conforte par ces variations sur l'URL qui masquent les serveurs gouvernementaux un certain potentiel fédérateur.

Malgré les tensions territoriales résiduelles ou latentes qui diffèrent le lancement de l'Atelier International du Grand Paris, la donne métropolitaine se décentre sur le site de la consultation ${ }^{26}$ : après les deux projets politiques en confrontation, ce sont donc dix projets architecturaux en compétition qui sont donnés à voir ensemble. La reprise énonciative de la consultation internationale sur le site de la Cité Chaillot ${ }^{27}$ adapte dès lors la métropole au régime de l'exposition.

Dans cet axe de discursivités prétendant à une expertise métropolitaine, la documentation accessible d'un savoir encrypté accompagne la vision des projets politiques et la signature de grands architectes leur porte caution. D'ores et déjà, la plus-value symbolique attendue de ces développements est celle qui octroie aux futures infrastructures une valeur d'emblèmes de la métropole à venir. Leurs images de synthèse et modèles à échelle sont censés opérer la rencontre des grands invariants politiques précédemment cités avec d'autres imaginaires forts dans la discursivité du monde contemporain : par le haut, les topoi 
de l'écologique et du numérique s'incorporent ainsi au complexe discursif métropolitain comme moteurs utopiques des chantiers innovants.

\section{L'entrelacs vernaculaire : les prises de parole ordinaires et les territoires vécus}

La configuration discursive précédemment décrite rend difficile la prise de parole des habitants de la région parisienne au sujet de leur devenir métropolitain. En effet, les projections politiques, urbanistiques ou architecturales de la métropole parisienne reposent sur une conception stratégique du territoire comme « lieu propre » qui demeure inaccessible depuis le niveau « tactique » de ses habitants ${ }^{28}$. En l'état actuel, les postures discursives qui sont réservées aux habitants de la métropole restent les suivantes : d'une part, les clivages politiques entre les projets existants convoquent avant tout une figure de l'électeur apportant (ou non) son soutien à un élu délégataire du devenir métropolitain ; d'autre part, les multiples propositions architecturales préfigurent une posture contemplative de spectateur, voire de futur habitant des maquettes exposées moyennant une capacité d'auto-projection.

Or, au-delà du « pour ou contre » de l'opinion ou des « livres d'or » des expositions, l'enjeu d'une prise de parole active des habitants est celui de donner aux projets métropolitains un ancrage vernaculaire. Du point de vue des discursivités, nous désignons par vernaculaire cette parole polymorphe et fluctuante que les dispositifs du Web contemporain sollicitent aux internautes; de ce fait, " elle ne constitue pas un discours coordonné ou téléologique et se caractérise par son abondance et sa polyphonie énonciative ${ }^{29}$.
Comme le souligne Robert Glenn Howard, le vernaculaire "réfère aux formes et pratiques culturelles non-institutionnelles $\gg{ }^{30}$. Pour Anouk Bélanger, dans l'imaginaire urbain contemporain, le vernaculaire joue un rôle de contrepoint dialectique par rapport au « spectaculaire » privilégié et investi institutionnellement ${ }^{31}$. Cet enracinement dans l'expérience quotidienne des territoires voués à devenir métropole se donne à lire sur le web de manière encore officieuse et fragmentaire, aux marges des projets estampillés par le pouvoir officiel comme discours circulants.

À ce stade de concertation prospective, sur des centralités vernaculaires telles que les forums de discussion ou les sites de réseaux sociaux, la parole des habitants parle encore un langage antémétropolitain : ses vues territoriales sont encore celles qui reproduisent les distances symboliques qu'entretiennent « Paris » et «La Banlieue », ou encore celles que revendiquent un attachement au « 92 », « 93 », « $94 »$, « 77 » comme espaces investis d'une valeur aussi identitaire qu'identificatoire. En témoignent de nombreux groupes sur Facebook que les habitants-internautes rejoignent, à l'image de «Banlieue 92, on est combien? Montrons notre force !!!!!!!!!! », « Banlieue Nord (92-93-95) », « Paris Banlieue Sud »32. Jouant sur certaines 'marginalités' stéréotypiques attribuées aux jeunes banlieusards, ces regroupements affichent des tensions symboliques et sociales qui auraient toute leur place dans un espace de débat métropolitain. La portée de ces rassemblements reste néanmoins limitée à un acte de visibilité en ligne, difficilement reconnaissable d'un point de vue institutionnel en tant que public agissant.

Sur les forums de discussion, ces tensions se font 
plus explicites et confrontent le projet de métropole à une expérience symbolique violente : «Les solutions existent mais elles dérangent. Faire le Grand Paris, faire exploser les banlieues et mélanger les gens socialement très vite dans les beaux quartiers... Les ghettos des classes privilégiées sont vus de la Courneuve, insupportables, car on sait dès le départ que les dés sont pipés d'avance juste pour quelques km de distance $»^{33}$, poste un internaute sur Discutons.org. D'autres commentent sur Forum-Fr les images des maquettes : «Paris ne sera plus Paris? », "J'espère que cette fiction sera réalité $»^{34}$.

En attendant la réappropriation discursive de la métropole par les habitants, les mises-en-récit de films comme Neuilly sa mère ou Tout ce qui brille suscitent aussi des prises de parole qui annoncent de manière oblique des futurs débats fondés sur l'expérience du métropolitain. « Moi qui habite à Neuilly sur Seine, qui a habité pendant 14 ans dans un quartier défavorisé, proche du quartier ZUP... Et qui déteste tous ces gamins bourgeois de Neuilly sur Seine... Je me ferais un plaisir d'aller voir ce film $»^{35}$ témoigne une internaute de « Melun (77) » au sujet du premier film; «Accumulation de clichés de gosses de riches + des clichés de gosses de pauvres », réplique un internaute disant habiter «Sankt Parisbourg $»^{36}$. Au prisme de Tout ce qui brille, un «allocinéen » exprime : «Ce film met une fois de plus en opposition 2 milieux sociaux différents. Mais on évite les caricatures des fameuses banlieues parisiennes $»^{37}$. En deçà d'une pensée systémique, émergeant du « monde vécu $»^{38}$ des territoires de la région métropolitaine ou en réaction à la médiatisation progressive des projets, ces prises de parole traitent déjà de questions métropolitaines en adoptant un point de vue situé, non surplombant.

Le cantonnement relatif de la métropole aux constructions d'un langage technocratique que les couches vernaculaires peinent à s'approprier constitue l'une des limites actuelles des projets proposés : la reconnaissance et assimilation de tels discours par les habitants représentent un enjeu de taille et c'est à ce titre que des sites Web ad hoc ont été mis en place pour impliquer la population francilienne concernée. À terme, la possibilité d'une réécriture vernaculaire de la métropolisation suppose un travail de fond sur la mémoire culturelle de ces territoires régionaux, dont les reliefs et frontières font l'objet de connotations sociales denses. Néanmoins, à ce stade des projets métropolitains, leur déploiement sur Internet relève davantage d'un travail de préparation à la concertation politique que d'une véritable réceptivité à des prises de parole partiellement déléguées.

\section{Des dénégations aux délégations de la parole : l'internaute et la métropole}

Lorsqu'elle n'est pas canalisée par un milieu " associatif $~^{39}$ assumant le rôle de porte-parole, la parole vernaculaire reste souvent cantonnée dans les blogs ${ }^{40}$, forums et sites de réseaux sociaux. D'un point de vue communicationnel, ces paroles ordinaires d'internautes-habitants présentent un intérêt croissant pour les acteurs politiques contemporains : elles supposent un feedback pouvant être reconnu tantôt comme une force de proposition utile (dans la veine du crowdsourcing), tantôt comme un indicateur chiffré de performance en termes de notoriété.

Là où au tournant du siècle, un Web institutionnel 
(celui des Webmasters d'institutions publiques et privées) se distinguait nettement d'un Web vernaculaire (celui des internautes autodidactes créant leurs pages personnelles), ces écarts tendent à se réduire sur le Web contemporain en vertu de formes techno-sémiotiques communes.

\section{Paroles à apprendre ou à prendre : des appa- reillages de la sollicitation}

En vertu de l'idéologie professionnelle qui a systématisé l'incorporation de modules de « commentaires », « notations » et boutons de « partage » aux dispositifs d'un Web dit « participatif», les formes des écritures s'articulent, facilitent la circulation du discours et entretiennent l'attente contemporaine selon laquelle l'internaute doit se voir déléguer des « opérations productives » sur les sites des secteurs aussi divers que la culture, la politique ou la consommation. Au sujet de la métropole, dans cette tendance « participative », le site « Le Grand Paris » ${ }^{41}$ illustre de manière exemplaire les prétentions mais aussi les limites de cette démarche.

Lancé par le SIG le 23 novembre 2009 à l'adresse http://www.mon-grandparis.fr, le site prétend s'inscrire parmi les « espaces privilégiés [qui] permettent aux Franciliens de donner leur avis et de s'approprier le Grand Paris ». Deux objectifs officiels émergent : «faire comprendre les enjeux $d u$ Grand Paris et recueillir les contributions des internautes $\gg^{42}$. Le dispositif est censé déployer et articuler auprès des internautes une double visée pédagogique et 'participative' sur le débat métropolitain.

Quant à la visée pédagogique, cette prétention qui va d'un faire-connaître à un faire-comprendre le projet du Grand Paris mobilise deux temps : un premier temps publicitaire où des bannières disposées sur des médias français en ligne ont parié sur une visibilité potentielle du projet à partir de l'autopromotion du site; dans un second temps, moyennant le trafic généré par cette campagne de lancement et ses retombées médiatiques, Mongrandparis.fr entend faire comprendre le projet.

En suivant le logo et son slogan «Le Grand Paris, une grande idée qui a besoin des vôtres » qui font partie du branding éditorial, l'exposition de la « grande idée » du projet concerne quelques textes manifestant l'ambition présidentielle mais repose essentiellement sur une scénographie 'testimoniale' qui parle du Grand Paris sous forme de vidéos. Des spots mettant en scène une 'diversité représentative' des publics franciliens ciblés déclinent les « thèmes » de la métropolisation avec des messages euphoriques simplifiés : dans la métropole des pôles, Saclay sera la « Silicon Valley » et le «métro fera le tour des banlieues »; aux «tours inhumaines » se substitueront les « meilleurs architectes» pensant à la «vie collective » des nouveaux quartiers ainsi qu'à «préserver les espaces verts »; enfin, « la culture va pousser partout ». Nulle mention du projet 'opposé' 43 Paris Métropole qui développe sa propre pédagogie audiovisuelle du « Faire métropole $\aleph^{44}$ sur le site de partage vidéo Dailymotion.

À chacun des « thèmes » métropolitains (compétitivité, transports, logement, culture) que l'internaute peut 'apprendre' du site se succède une série d'injonctions à la prise de parole qui font la spécificité du site « Le Grand Paris ». Audelà de la compréhension approfondie du projet, 
une enveloppe énonciative prétend « recueillir les contributions des internautes » en jouant d'une adresse directive : "Venez prendre part au débat sur le Grand Paris », "Venez, vous aussi, défendre votre vision du Grand Paris», «Découvrez déjà plus de 2000 idées et commentaires », «Vous aussi, contribuez $»^{45}$. La rhétorique du 'participatif' est celle qui métaphorise les « figures contributives ${ }^{46}$ disponibles pour encourager la productivité en volume des autopublications. Loin des vieux questionnaires, les formulaires informatiques ajoutent la possibilité de connoter une expression personnelle dans leur captation de paroles : «Mon-grandparis $»^{47}$ comme « MySpace » ou «YouTube » suggèrent des possessifs projectifs qui font de la « création » d'un compte, et de la saisie de « commentaires » des gestes valorisateurs, investis d'un imaginaire de « bulles » d'oralité là où il n'y a que champs de scripturalisation.

Ce qui est écrit et tracé, « recueilli » ou « recensé » n'est donc pas pour autant ni 'dit' ni 'entendu'. En effet, la 'participation' ne se fait que dans le cadre des « thèmes » précités, ceux-ci sont déclinés en « idées $»^{48}$ » que le dispositif du SIG prédécoupe, lesquelles sont « notées » ou « commentées » par les internautes. Cette configuration discursive métaphoriquement 'égalitaire' mais éditorialement 'dissymétrique' évoquerait une participation du type : «Le Grand Paris, une grande idée fragmentée en sous-idées qui ont besoin de vos commentaires et vos notes $\gg$. La position vernaculaire ainsi préfabriquée vise avant tout à entretenir un imaginaire relationnel de la proximité ou de l'écoute, la où les « appels à initiatives » ${ }^{49}$ adoptent des formes peu médiagéniques ${ }^{50}$ et les chats avec des responsables politiques ne marquent que d'éphémères rencontres. En contraste avec les formes basiques et les cercles limités du forum de ParisMétropole.fr ${ }^{51}$, la dynamique inter-discursive de mon-grandparis prétend institutionnaliser le vernaculaire en effectuant un cadrage fort : celui d'un appareillage qui centralise, sollicite et cite les internautes moins comme des forces de proposition délibératives que comme des vecteurs quantifiables de « priorisation » et de «popularité » autour des « idées les mieux notées ».

Autour de la métropolisation, la mise à profit de ces dispositifs suppose une adaptation du discours politique à des formes censées impliquer les internautes et leur déléguant la parole à des degrés divers. En termes de communication sur le projet, les objectifs politiques sous-jacents à ces pages $a d h o c$ - la réduction de l'opacité, recherche de la polyphonie, l'exploitation de la légitimité - tendent à mettre les rhétoriques du 'participatif' et les imaginaires 'démocratiques' en tension avec la forme même des appareillages techniques proposés à l'expression.

\section{Des métropoles non-dites sur le Web ? ouver- tures non-institutionnelles}

En dehors des sites consacrés à la promotion des projets métropolitains - lieux d'une lecture politisée et/ou d'une fantasmatique remontée de paroles citoyennes - les axes inter-discursifs étudiés nous invitent à penser au problème de la métropole parisienne en fonction de deux dichotomies principales qui éclatent les discours et creusent leurs écarts : entre surplomb et localisation se bifurquent les échelles du territoire comme lieu propre ; entre spectaculaire et vernaculaire se brouillent les marqueurs du devenir 
métropolitain.

Aux marges des constructions discursives institutionnelles qui figent l'internaute dans des positions d'électeur, spectateur, usager ou citoyen, c'est la figure de l'habitant qui est mise à l'écart. Quelques formes du Web susceptibles de projeter sur l'internaute certaines des pratiques du futur 'habitant métropolitain' échappent sans doute à la reconnaissance des mots-clés grand ou métropole, en faveur d'une certaine fluidité trans-territoriale : celle des géographies symboliques potentiellement présentes dans l'extension « . paris $\rangle^{52}$. Du côté des guides de sorties nocturnes, une métropole latente s'insinue entre les agendas et les cartes $^{53}$, dont la mise en visibilité pourrait à terme décliner des 'scènes' sous-culturelles et des effets de mode. Au sein de sites géographiques ou de réseaux sociaux de proximité ${ }^{54}$, une logistique d'échanges et activités proposées connote une convivialité pertinente dans la sociabilisation de 'nouveaux' parisiens. Enfin, sur des sites associatifs ou de solidarité se donnent à voir des causes ponctuelles à rejoindre ${ }^{55}$, lesquelles canalisent des militances ou des engagements aptes à faire l'objet d'un travail d'advocacy remontant des intérêts au-delà des faits de parole. Au quadrant d'une localisation avec des surplombs occasionnels ou d'une spectacularisation noninstitutionnelle, un travail de longue haleine sur l'axiologie des représentations de la « présence » et de la « fonction de Paris ${ }^{56} »$ s'insinue. Entre les médiations techniques qui informent les écritures et les imaginaires territoriaux qui hantent les discours, c'est en termes d'activités et d'affinités que les réseaux devraient démarquer les lieux de la grande métropole.
$\mathrm{N} \cdot \mathrm{O} \cdot \mathrm{T} \cdot \mathrm{E} \cdot \mathrm{S}$

1. Eliseo Véron, La sémiosis sociale : fragments d'une théorie de la discursivité, Saint Denis, PUV, 1987, p. 132.

2. Dominique Pagès, «L'inscription du projet de Métropole de Paris dans l'espace public numérique: émergences et ouvertures ", in Quaderni, ${ }^{\circ} 71$, Hiver 2009/2010, pp. 75-84.

3. Voir, sur ce point, l'entretien avec Pierre Mansat dans ce volume.

4. Michel de Certeau, L'Invention du quotidien, 1. Arts de faire, Paris, Gallimard, 1990. p. XLVI.

5. http://www.mon-grandparis.fr/

6. http://www.mon-grandparis.fr/le-grand-paris

7. http://www.mon-grandparis.fr/la-consultation

8. http://www.parismetropole.fr/

9. http://www.iledefrance.fr

10. http://www.iledefrance.fr/missions-et-competences/le-conseil-regional/le-fonctionnement-du-conseilregional/lexecutif/

11. http://www.legrandparis.culture.gouv.fr/

12. http://blogs.senat.fr/grandparis/

13. http://www.paris.fr/portail/politiques/Portal. lut?page_id=8682; http://www.paris.fr/portail/accueil/ portal.lut?page_id $=8700$

14. http://www.pierremansat.com/

15. http://www.pierremansat.com/article-7367686. html

16. Alice Krieg-Planque, La notion de "formule" en analyse du discours. Cadre théorique et méthodologique, Besançon, Presses Universitaires de FrancheComté, 2009.

17. Marie-Eve Thérenty, La littérature au quotidien : poétiques journalistiques au XIXe siècle, Paris, Seuil, 2007, p. 78.

18. Les sites de campagne de Roger Karoutchi et 
Valérie Pécresse investissaient ce créneau discursif pour 2008 ou 2010 : http://www.debat-grandparis. com ; http://www.pecresse-iledefrance.fr/search/node/ grand $\% 20$ paris

19. http://www.apur.org/metropole

20. http://www.iaurif.org/debats-enjeux/les-grandsparis-dune-metropole-durable.html

21. http://www.iau-idf.fr/fileadmin/user_upload/Enjeux/Paris_metropole/Biblio_GrandParis.pdf

22. http://www.iau-idf.fr/fileadmin/user_upload/Enjeux/gd_projets_urbains/Colloque_22-23_nov_2007/ actes_colloque_grands_projets_urbains.pdf

23. http://www.iau-idf.fr/debats-enjeux/paris-metropole/groupes-de-travail/projets-metropolitains.html

24. http://www.sdrif.com/

25. http://www.legrandparis.culture.gouv.fr

26. http://www.legrandparis.culture.gouv.fr/equipes

27. http://www.citechaillot.fr/exposition/expositions temporaires.php?id $=80$

28. Michel de Certeau, L'Invention du quotidien, 1. Arts de faire, op. cit., pp. 57-63.

29. Claire Burlat et Gustavo Gomez-Mejia, « La crise aux prises du vernaculaire : paroles d'internautes », Communication et Langages, ${ }^{\circ} 162$, décembre 2009 , pp. 91-106.

30. Robert Glenn Howard, "The Vernacular Mode: Locating the Non-Institutional in The Practice of Citizenship" in R. Asen et D. Brower (éd.), Public Modalities, New York, University of Alabama Press, 2010, 240-261.

31. Anouk Bélanger, «Montréal vernaculaire/Montréal spectaculaire : dialectique de l'imaginaire urbain », Sociologie et sociétés, vol. 37, n 1, 2005, pp. 13-34.

32. Pages « groupes» homonymes accessibles par le moteur de recherche de Facebook.com

33. http://www.discutons.org/Sujet-de-Discussion35910-0-asc-20.html

34. http://www.forumfr.com/sujet272186-le-grand- paris-dans-vingt-ans-en-images.html

35. http://www.forumfr.com/news305752-neuilly-samere.html

\section{Ibid.}

37. http://www.allocine.fr/communaute/forum/message_gen_nofil $=596151 \&$ cfilm $=\&$ refpersonne $=\&$ car ticle $=\&$ refserie $=\&$ refmedia $=\&$ page $=2 . \mathrm{html}$

38. Cf. Jürgen Habermas, Théorie de l'agir communicationnel, tome 2, Fayard, Paris, 1987, chap. VI.

39. Voir par exemple le site de l'Association Grand Paris, comptant 589 « fans » sur sa page Facebook. http://www.associationgrandparis.fr/

40. Dans la mesure où certains blogs se spécialisent, leurs auteurs acquièrent ou prétendent acquérir une certaine visibilité institutionnelle : http://parisbanlieue.blog.lemonde.fr ; http://parisinternational. blogspot.com/

41. http://www.mon-grandparis.fr

42. http://www.mon-grandparis.fr/le-grand-paris

43. Voir les contributions de Frédéric Gilli et de Dominique Pagès au présent numéro thématique.

44. http://www.dailymotion.com/Faire_Metropole

45. Une rhétorique semblable est à observer sur le site du Nouveau Centre : http://www.grandparis2014. com/

46. Emmanuelle Lambert et, Jean-Thierry Julia, Énonciation et interactivité : du réactif au créatif, Communication et langages, $n^{\circ} 137$, p. 40.

47. L'adresse prévisible http://www.mongrandparis. com, hébergeait un « blog citoyen et indépendant sur Paris et sa région » aujourd'hui abandonné.

48. http://www.mon-grandparis.fr/participer-au-debat

49. http://www.parismetropole.fr/component/content/ category/30-appel-a-initiatives-paris-metropole

50 . Le terme « médiagénie » désigne pour une représentation sa «possibilité de se réaliser de manière optimale en choisissant le partenaire médiatique qui 
lui convient le mieux ». Cf. Philippe Marion, « Narratologie médiatique et médiagénie des récits » in Recherches en communication, $\mathrm{n}^{\circ} 7$, Louvain, UCL, 1997, p. 86.

51. http://www.parismetropole.fr/forum

52. http://www.soutenonspointparis.fr/

53. http://www.parisnightlife.fr/ ; http://www.parisbouge.com/ ; http://www.spectaculaire.com/ ; http:// www.vodkacoca.com/lieux/

54. http://des-gens.net/; http://paris.peuplade.fr ; http:// paris.onvasortir.com/ ; http://paris.voisineo.com/

55. http://www.gambetta-village.com/ ; http://www. canalmarches.org/; http://www.soutenonspointparis. fr/content/la-liste-des-signataires ou d'autres revendications ponctuelles déclinant les types de http:// causes.org

56. Paul Valéry, «Fonction de Paris » et « Présence de Paris » [1927-1937], in Regards sur le monde actuel, Paris, Gallimard, 1945.

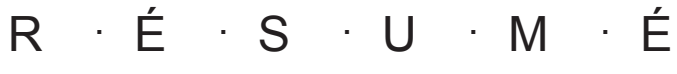

Le présent article étudie les formes dominantes de représentation de la métropole parisienne sur l'Internet contemporain. Autour des projets du Grand Paris et de Paris Métropole, l'approche privilégiée est celle d'une analyse interdiscursive menée sur un corpus de sites Web. Dans un spectre qui va de l'institutionnel au vernaculaire, en passant par le journalistique et l'académique, ces sites cristallisent des zones de rencontre et des écarts discursifs au sujet de la métropole en devenir. La question de la circulation des discours sur la métropolisation est ainsi mise en relation avec les logiques sociales des acteurs mais aussi avec les formes techniques des dispositifs du Web.

\section{Summary}

This article examines the dominant forms of representation of Metropolitan Paris on the contemporary Internet. An interdiscursive approach is adopted by analysing a corpus of websites related to the Grand Paris and Paris Métropole initiatives. Institutional, vernacular, journalistic and academic discourse types reveal a set of gaps and meeting-points with varying views on the metropolisation process. The study of such on-line discursive circuits thus requires to take into account the interests of their authors, but also to the technical features of Web devices. 